\title{
TEMA 17-2014: LA VÍA DE GONTACTO: ¿EL FUTURO DE LOS ANTICOAGULANTES?
}
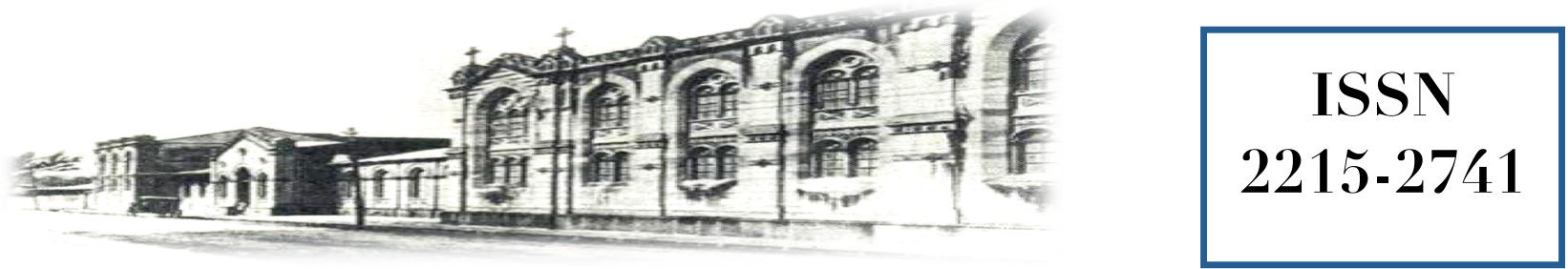

Haspital San quan de Dias. San Jasé. Casta Rica. Fundada en 1845

$\begin{array}{ll}\text { Recibido: } & 28 / 07 / 2014 \\ \text { Aceptado: } & 26 / 11 / 2014\end{array}$

Max A. Méndez López ${ }^{1}$

${ }^{1}$ Médico Especialista en Hematología. Servicio de Hematología Hospital Dr. R.A Calderón Guardia. Correo electrónico: maxmendez@,runbox.com

\section{RESUMEN}

Durante décadas el desarrollo de nuevos anticoagulantes se ha basado en la modificación de fármacos o moléculas previamente disponibles. La poca oferta ha ocasionado que actualmente sea aceptado no administrar anticoagulantes cuando existe una indicación clara de anticoagulación, incrementando así el riesgo de extensión del fenómeno trombótico, el síndrome postrombótico, la discapacidad a largo plazo y las recurrencias, que en conjunto causan más morbilidad y mortalidad que el sangrado secundario a sobreanticoagulación. Los nuevos anticoagulantes orales han ampliado la cantidad de pacientes potenciales, sin embargo existen más opciones viables a mediano plazo que pueden cambiar la anticoagulación tal y como la conocemos hoy en día. Se resume la evidencia disponible en cuanto a la utilización del Sistema de Contacto de la coagulación como modelo para el desarrollo de futuras opciones terapéuticas y sus implicaciones futuras.

\section{PALABRAS CLAVE}

Anticoagulación. Sistema de Contacto. Factor XII.

\section{ABSTRACT}

For years, the development of new anticoagulant drugs has been based on modifications of previously available compounds. Currently, it is widely accepted to withhold the administration of anticoagulants even in the presence of an indication of anticoagulation, increasing the morbidity, long-term disability and relapses, which together account for more mortality than bleeding as a secondary effect. The novel oral anticoagulants have broadened the number of potential candidates; however, more options are foreseen in the future that can change clinical anticoagulation as it is prescribed today. We will summarize current knowledge on the coagulation Contact System and future therapeutic options. 


\section{KEY WORDS}

Anticoagulation. Contact System. FXII.

\section{INTRODUCCIÓN}

El desarrollo de nuevos anticoagulantes ha sido lento comparativamente con el de otras drogas como antibióticos o quimioterapéuticos. El sangrado y la incertidumbre que ha creado la necesidad o no de un método de monitoreo han sido en gran parte responsables de que la diseminación de los nuevos anticoagulantes orales (NAO) no haya sido generalizada. Aunque recientemente se ha ampliado el número de potenciales candidatos a anticoagulación con la aparición de los $\mathrm{NAO}$, las opciones actuales son limitadas al no incluir aquellos pacientes con contraindicaciones para anticoagulantes pero con indicaciones absolutas para anticoagulación. Si se analiza brevemente el modelo de coagulación basada en células (El Sistema), o la coagulación desde el punto de vista proteico (La Cascada) es fácil identificar la trombina, el FX y el FII como los blancos recurrentes para el desarrollo de nuevos anticoagulantes orales y/o parenterales, pero aunque el espectro de posibles candidatos para el desarrollo de nuevos compuestos es amplísimo, no todos cumplen con las características necesarias para convertirse en un fármaco comercial.

El objetivo de esta revisión es proveer una actualización en la vía de contacto como opción terapéutica a mediano plazo, una vía que puede cambiar lo que actualmente conocemos como anticoagulación.

\section{DISCUSIÓN}

\section{Historia}

En 1955, Ratnoff y Colopy estudiaron un paciente a quien se le documentó tiempos de coagulación prolongados en la rutina preoperatoria, sin manifestación clínica alguna. Curiosamente el paciente había sido sometido a dos procedimientos quirúrgicos previos sin ninguna complicación $^{(1)}$. Ambos autores lograron identificar la proteína faltante como factor XII (FXII) y el hecho de que John Hageman no presentara sangrados sugería que la actividad de dichas proteínas era en forma de precursores inactivos ${ }^{(2)}$. John Hageman falleció, irónicamente, de embolismo pulmonar masivo teniendo deficiencia en una proteína de la coagulación ${ }^{(3)}$. El sistema de contacto ha sido incluido históricamente dentro de la vía intrínseca de la cascada y más recientemente en la primera fase del sistema de coagulación. El FXII juega un papel fundamental aquí; sin embargo, el fenotipo contrastante entre los pacientes asintomáticos deficientes en FXII y el sangrado difícil de controlar en los hemofílicos deficientes de FVIII o FIX cuestionó durante muchos años que la coagulación actuara como una cascada de activación enzimática ordenada, en la cual una deficiencia del FXII causaría un bloqueo absoluto en la producción de trombina por la vía intrínseca. A pesar de ser una cascada activada por proteólisis, Osterud demostró que el complejo FT:FVIIa era capaz de activar al FIX sin necesidad de activar al FXII y FXI, con lo que el modelo ordenado y secuencial quedó en duda ${ }^{(4)}$.

\section{Bioquímica del Factor XII}

El sistema de contacto está formado por el kininógeno de alto peso molecular (HK, PM: 110.000 Daltons), prekalikreína (PK, PM: 88.000 Daltons), FXII (PM: 80.000 Daltons) y FXI (PM: 143.000 Daltons $)^{(5)}$. El FXII consta de 596 aminoácidos y está formado por una cadena liviana de 243 residuos y una pesada de 353 residuos.

La lista de activadores del FXII crece rápidamente e incluye una gran variedad de materiales biológicos, membranas y sustancias endógenas ${ }^{(6)}$; sin embargo, es independiente del factor tisular (FT). El FXII es una glicoproteína de cadena única que circula en plasma a concentraciones de $375 \mathrm{nM}(30 \mu \mathrm{g} / \mathrm{ml})$, con una vida media de 50-70 horas ${ }^{(7)}$. Es activado por dos mecanismos: por corte enzimático en la posición R353-R354 o por autoactivación al unirse a superficies cargadas negativamente (Figura 1), provocando cambios conformacionales diferentes en cada caso ${ }^{(8)}$.

El FXII se activa para formar ( $\alpha$-FXIIa); de igual forma, la PK es activada por el $\alpha$-FXIIa, que actúa reciprocamente activando más FXII y liberando bradikinina a partir del $\mathrm{HK}^{(7,9)}$. La bradikinina es el ligando del receptor de proteinas G kinina B2 y actúa como un mediador inflamatorio, incrementando la permeabilidad vascular al estimular la producción de óxido nítrico por parte de las células endoteliales ${ }^{(10)}$. 

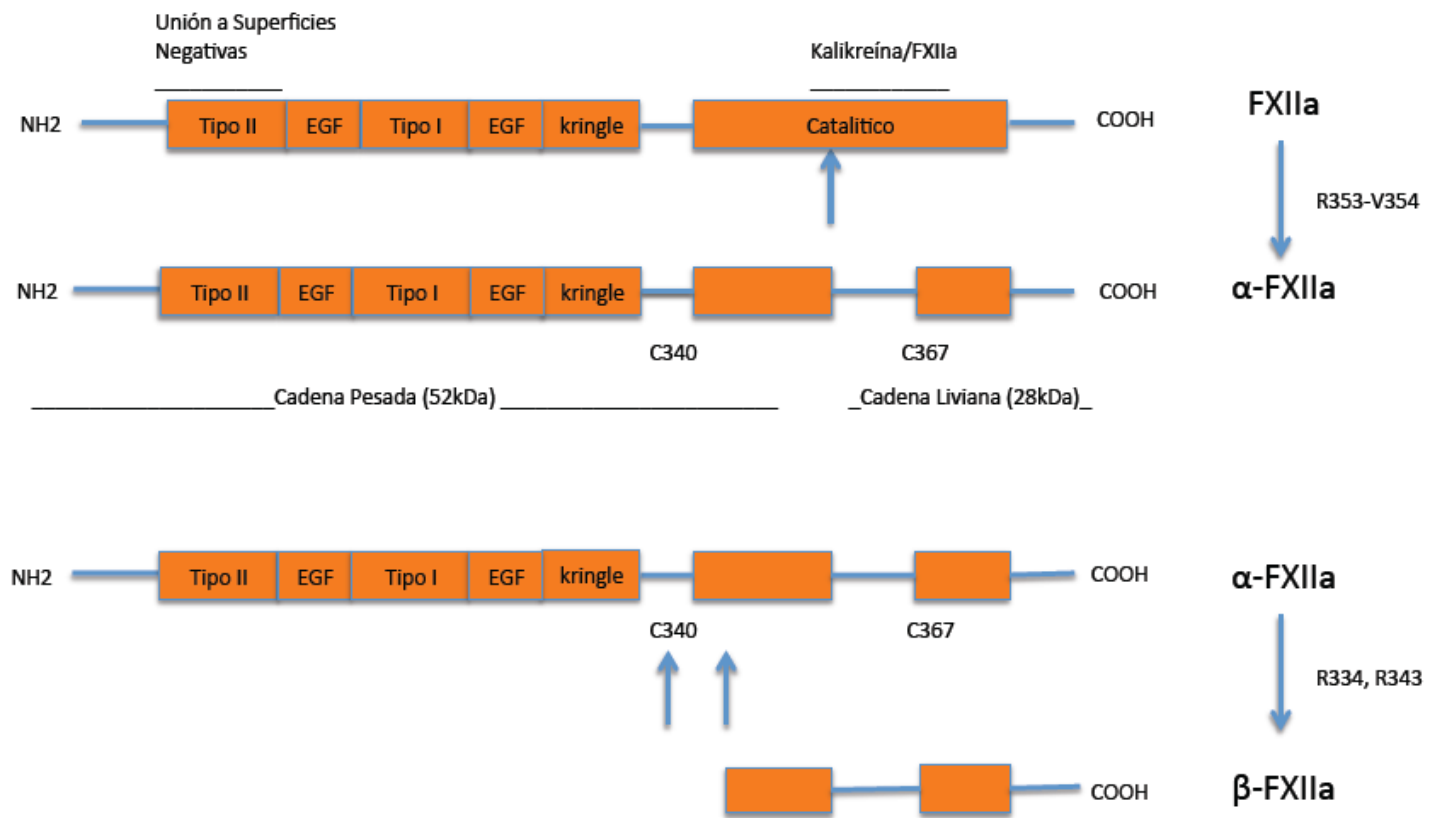

Figura 1. Estructura y Activación del FXII

El corte enzimático en la posición R353-R354 activa el FXII, formando ( $\alpha$-FXII). Un Segundo corte en C340 y C367 mediado por la kalikreína produce $\beta$-FXIIa, el cual tiene pobre adherencia a superficies cargadas negativamente debido a la falta de la mayoría de su cadena pesada.

El corte de $\alpha$-FXIIa mediado por la kallikreína produce Factor $\beta$ XIIa ( $\beta$-FXIIa), una forma enzimática de la cadena liviana que activa el complejo macromolecular Clqrs del complemento, provocando activación de la cascada clásica del complemento y producción de kalikreína ${ }^{(1)}$. El C1-inhibidor es la proteína inhibidora más importante de PK y del FXII ${ }^{(12)}$; además, el inhibidor de la proteína de soya "corn tripsin inhibitor" (CTI) ha demostrado inhibir la vía de contacto in vitro ${ }^{(13)}$. En resumen, las consecuencias de la activación del FXII se observan en tres vías: coagulación, inflamación y complemento (Figura 2), produciendo fibrina, activación del Clqrs y liberación de bradikinina.

\section{Papel del FXII in vivo}

Las actividades biológicas del FXII aún se encuentran bajo investigación. Se ha sugerido que los polifosfatos secretados por plaquetas son activadores in vivo del FXII 14. Los polifosfatos son cadenas largas de fosfatos cargados negativamente, a quienes algunos autores consideran el punto de interacción entre la hemostasia primaria y secundaria ${ }^{(11)}$. También se ha demostrado que los polifosfatos plaquetarios promueven la coagulación sin intervención del FXI 15.

Datos recientes han cuestionado la validez de los estudios con los polifosfatos, fallando al reproducir resultados previos ${ }^{(16)}$.

Al día de hoy el mecanismo de formación de los polifosfatos no ha sido elucidado y existe un debate activo sobre su importancia real en la coagulación $^{(17)}$. Es interesante que se ha demostrado que la activación del FXII mediada por los polifosfatos plaquetarios no conlleva una formación acelerada del trombo, sino un incremento en su estabilidad ${ }^{(18,19)}$. Igualmente, una unión zinc-dependiente del FXII a superficies cargadas negativamente descrita previamente podría explicar la unión al cluster de fosfatos cargados negativamente ${ }^{(20)}$ y posiblemente el mecanismo de unión de ambas moléculas $^{(21)}$. Teóricamente, el bloqueo de esta interacción por fosfatasas o hidrolasas podría ser un mecanismo regulador de la activación del FXII y por ende de la formación del trombo.

Curiosamente algunos activadores del FXII como el lipopolisacárido bacteriano (LPS) o el $\beta$ amiloide no evocan una respuesta procoagulante 
mediada por el FXII, sino que estimulan la señalización por la vía kalikreína-kinina, reforzando la idea de que tanto el $\alpha$-FXIIa como

el $\beta$-FXIIa median vías de señalización $\operatorname{distintas}^{(22,23)}$.

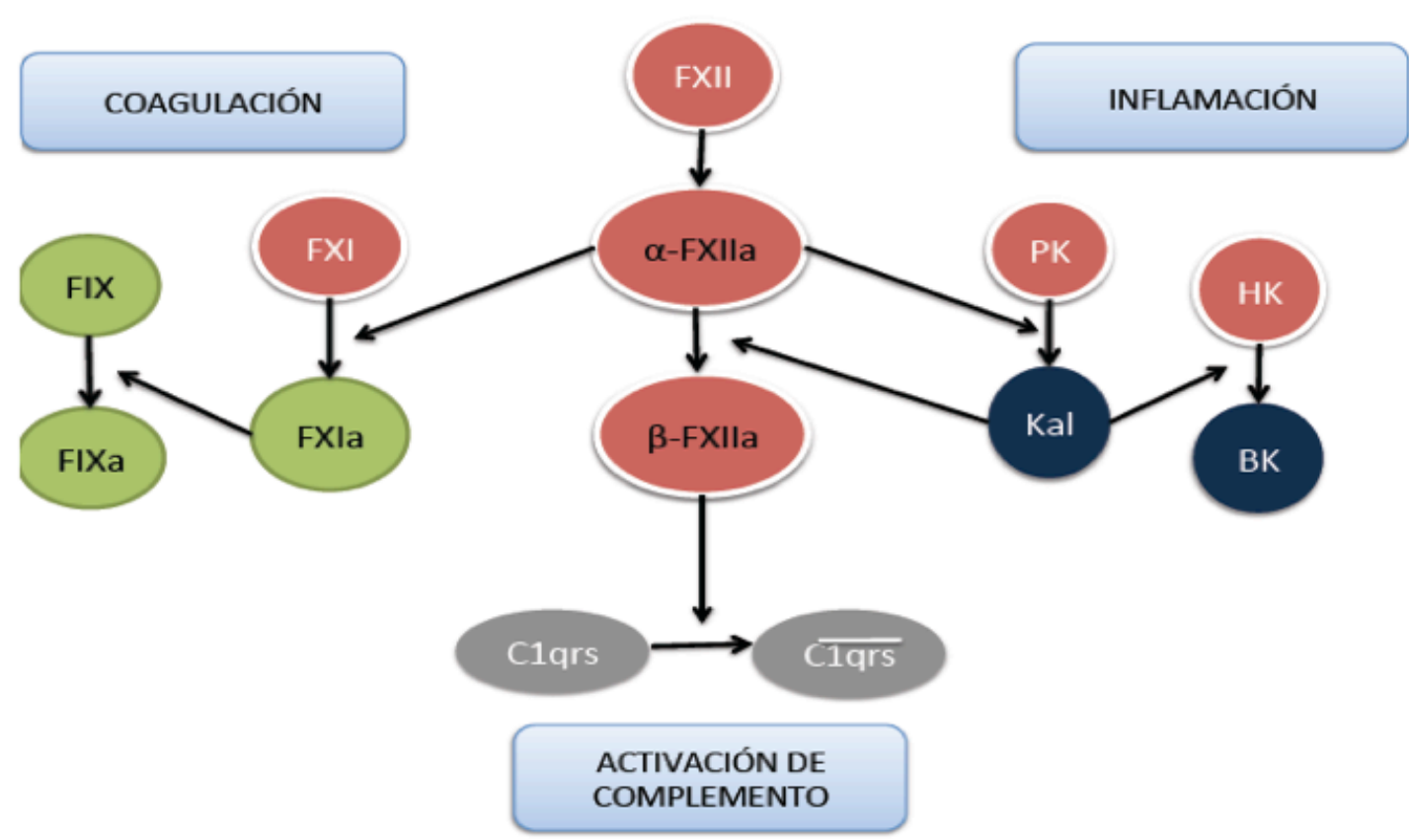

Figura 2. Interacciones del Sistema de Contacto.

El sistema de contacto interactúa con la vía del complemento (gris) y las vías inflamatorias (azul).

Los estudios en ratones $\mathrm{FXII}^{-/-}$han demostrado ausencia del FXII plasmático, ausencia de trombofilia, ausencia de alteraciones en la fibrinólisis y además ausencia de hemorragia; es decir, un fenotipo idéntico a los humanos deficientes en FXII ${ }^{(19)}$. Los ratones sufrieron un defecto severo en la formación y estabilización del trombo rico en plaquetas que revirtió con la administración intravenosa de FXII, sugiriendo por primera vez que distintas vías eran las responsables de la hemostasia y de la formación patológica del trombo.

En condiciones de bajo flujo como el sistema venoso, el colágeno y la laminina producen trombos ricos en plaquetas mediados por la activación del FXII ${ }^{(24,25)}$ y en condiciones de alto flujo como el arterial se ha localizado el FXII en placas ateroscleróticas hacia sitios con mayor densidad de fibrina ${ }^{(26)}$, sugiriendo un papel predominante en la estabilización del coágulo. Es más, en un modelo de isquemia-reperfusión arterial se demostró que aquellos ratones con deficiencia del FXII sufrieron infartos cerebrales significativamente menores que los controles, y la reconstitución de sus niveles elevó la susceptibilidad a lesiones cerebrales isquémicas, indicando un efecto protector específico en la ausencia del FXII ${ }^{(27)}$.

Para hacer énfasis en este punto es importante recalcar que un inhibidor selectivo del FXIIa ha sido ensayado en ratones. Infestin4 unido a albúmina (rHA-infestin-4) se utilizó en un modelo de oclusión arterial cerebral, demostrando que el tratamiento de los ratones inhibió por completo el desarrollo de trombos con un riesgo bajo de sangrado ${ }^{(28)}$.

Igualmente, el rHA-infestin-4 actuó como un inhibidor reversible en un modelo de trombosis arteriovenosa en conejos ${ }^{(29)}$. Otro mecanismo alternativo ha sido dirigir anticuerpos monoclonales hacia la cadena pesada del FXII, que en primates ha demostrado reducir la formación de fibrina ${ }^{(30)}$. 
El uso de oligonucleótidos antisentido también fue explorado recientemente, con lo cual se logró bloquear la expresión del FXII y PK inhibiendo el desarrollo de thrombosis arteriales en ratones $^{(31)}$. De igual forma, la reducción de la expression del FXII y FXI en conejos redujo la incidencia de thrombosis venosa de catéter central $^{(32)}$.

En humanos el FXII fue considerado previamente como un predisponente para el desarrollo de enfermedad tromboembólica venosa, sin embargo su papel en pacientes con deficiencia homocigota de esta proteína demostró ser en el mejoror de los casos mínimo $^{(33)}$, lo que sugiere que la deficiencia de FXII en sí misma no es protrombótica. Se ha estimado la prevalencia de trombosis en pacientes deficientes de FXII en $2 \%{ }^{(34)}$.

\section{CONCLUSIONES}

A pesar de su papel controversial 60 años después de ser descubierto, el FXII sigue siendo explotado a diario en los análisis de coagulación de rutina como en el tiempo parcial de tromboplastina activada (TPTa).

Tras décadas de investigación, la función específica del FXII dentro del sistema del contacto es todavía incierta. Datos experimentales han demostrado que juega un papel de molécula orquestante, extendiendo señales a la vía del complemento, la vía inflamatoria por medio de bradikinina $y$ coagulación por medio del FXI.

Si bien el anticoagulante ideal está lejos de llegar, la vía intrínsica y en particular el FXII ofrecen un panorama prometedor a mediano plazo. El hecho de que los pacientes deficientes en FXII no presenten sangrado y que éste a su vez module la estabilización del trombo in vivo sugiere que un fármaco cuyo mecanismo de acción sea bloquear su activación o interacciones ofrecería mayor beneficio con menos riesgos que las opciones actuales y además podría ser utilizado en aquellas poblaciones con una indicación absoluta de anticoagulación pero con contraindicaciones para el uso de los anticoagulantes actuales.

\section{REFERENCIAS BIBLIOGRÁFICAS}

1. Rattnof OD Colopy J. A familial hemorrhagic trait associated with a deficiency of a clot-promoting fraction of plasma. J Clin Invest. 1955;34(4):602-613.

2. Schmaier A. The elusive physiologic role of Factor XII. J Clin Invest. 2008; 118:3006-3009.

3. Ratnoff OD Busse RJ Sheon RP. The demise of John Hageman. N Engl J Med. 1968;279:760-761.

4. Osterud B Rapaport SI. Activation of factor IX by the reaction product of tissue factor and factor VII: additional pathway for initiating blood coagulation. Proc Natl Acad Sci USA. 1977; 74:5260-5264.

5. Gailani D Renne T. Intrinsic Pathway of Coagulation and Arterial Thrombosis. Arterioscl Thromb Vasc Biol. 2007;27:2507-2513.

6. Maas C Oschartz C Renne T. The plasma contact system 2.0. Semin Thromb Hemost. 2011;37:375-381.

7. Colman RW Schamier A. Contact System: A vascular biology modulator with anticoagulant, profibrinolytic, antiadhesive and proinflammatory attributes. Blood. 1997;90(10):3819-3843.

8. Renne T. The Factor XII-driven plasma contact system. En Marder V, Aird W, Bennett $\mathrm{J}$ et al editors. Hemostasis and Thrombosis: Basic Principles and Clinical Practice. Lippincott Wilkins \& Williams. 2012.

9. Müller F Renne T. Novel roles for factor FXII-driven plasma contact activation system. Curr Opin Hematol. 2008; 15(5):516-521.

10. Leeb-Lundberg LM Marceau F MullerEsterl W Pettibone DJ Zuraw BL. Classification of the kinin receptor family: from molecular mechanisms to pathophysiologica consequences. Pharmacol Rev. 2005;57:27-77.

11. Müller F Mutch NJ Schenk WA et al. Platelet polyphosphates are proinflammatory and procoagulant mediators in vivo. Cell. 2009;139(6):1143-1156.

12. De Angostini A Lijnen HR Pixley RA Colman RW Schapira M. Inactivation of FXII active fragment in plasma: Predominant role of C1-inhibitor. $\mathrm{J}$ Clin Invest .1984;73(6):1542-1549. 
13. Nielsen VG. Corn Trypsin inhibitor decreases tissue-type plasminogen activator-mediated fibrinolysis of human plasma. Blood Coag Fibrinolysis. 2009; 20(3):191-196.

14. Smith SA Mutch N Baskar D Rohloff P Docampo R Morrissey J. Polyphosphate modulates blood coagulation and fibrinolysis. Proc Natl Acad Sci USA. 2006; 103(4):903-908.

15. Pui C Tucker EI Wong ZC et al. Factor XII promotes coagulation independent of FXI in the presence of long-chain polyphosphates. J Throm Haemost. 2013;11(7):1341-1352.

16. Faxälv L Boknäs N Ström JO et al. Putting polyphosphates to the test: evidence against platelet-induced activation of FXII. Blood. 2013;122(23): 3818-3824.

17. Maas C Renne T. Regulatory mechanisms of the plasma contact system. Thrombosis Res. 2012;129:S73-S76.

18. Pauer HU Renne T Hemmerlein B et al. Targeted deletion of murine coagulation factor XII gene-a model for contact phase activation in vivo. Thromb Haemost. 2004;92:503-508.

19. Renné T Pozgajová M Grüner S et al. Defective thrombus formation in mice lacking coagulation factor XII. J Exp Med. 2005;202(2):271-281.

20. Schousboe I Rojkjaer R. The surfacedependent autoactivation mechanism of factor XII. Eur J Biochem. 1997;243: 160-166.

21. Schousboe I. Pharmacological regulation of Factor XII activation may be a new target to control pathological coagulation. Biochem Pharmacol. 2008; 75:1007-1013.

22. Pixley R De La Cadena R Page J et al. The contact system contributes to hypotension but disseminated intravascular coagulation in lethal bacteremia: in vivo use of a monoclonal anti-FXII antibody to block contact activation in baboons. J Clin Invest. 1993;91:61-68.

23. Shibayama Y Joseph K Nakazawa Y Ghebreihiwet B Peerschke El Kaplan AP. Zinc-dependent activation of the plasma kinin-forming cascade by aggregated beta amyloid protein. Clin Immunol. 1999;90:89-99.

24. van der Meijden PE Munnix IC Auger
JM et al. Dual role of collagen in factor XII-dependent thrombus formation. Blood. 2009;114(4):881-890.

25. White-Adams TC Berny MA Patel I et al. Laminin promotes coagulation and thrombus formation in a FXIIdependent manner. J Thromb Hemost. 2010;8:1295-1301.

26. Konings J Govers-Riemslag JW Phillippou $\mathrm{H}$ et al. Factor XIIa regulates the structure of the fibrin clot independently of thrombin generation through direct interaction with fibrin. Blood. 2011; 118(14):3942-3951.

27. Kleinschnitz C Stoll G Bendszus M et al. Targeting coagulation factor XII provides protection from pathological thrombosis in cerebral ischemia without interfering with hemostasis. J Exp Med. 2006;203(3):513-518.

28. Hagedorn I Schmidbauer S Pleines I et al. Factor XIIa inhibitor recombinant human albumin Infestin-4 abolishes occlusive arterial thrombus formation without affecting bleeding. Circulation 2010;121:1510-1517.

29. Xu Y Quan-Cai T Castriota G Zhou Y Hoos L Jochnowitz N. FXIIa inhibition by infestin-4: in vitro mode of action and in vivo antithrombotic benefit. Thomb Haemost. 2014;111:694-704.

30. Matanof A Leung P Gailani A et al. Factor XII inhibition reduces thrombus formation in a primate thrombosis model. Blood. 2014;123(11):1739-1746.

31. Revenko A Gao D Crosby J et al. Selective depletion of plasma prekallikrein or coagulation FXII inhibits thrombosis in mice without increasing the risk of bleeding. Blood. 2011;118:5302-5311.

32. Yau J Liao P Fredenburgh J et al. Selective depletion of $f X I$ or $f X I I$ with antisense oligonucleotides attenuates catheter thrombosis in rabbits. Blood. 2014; 120:2102-2107.

33. Girolami A Randi ML Gavasso S Lombardi AM Spiezia F. The occasional venous thromboses seen in patients with severe (homozygous) FXII deficiency are probably due to associated risk factors: a study of prevalence in $21 \mathrm{pa}$ tients and review of the literature. $\mathrm{J}$ Thromb Thrombolysis. 2004;17(2):139143. 
34. Halbmayer WM Haushofer A Schön R et al. The prevalence of moderate and severe FXII (Hageman factor) deficiency among the normal population: evaluation of the incidence of FXII deficiency among 300 healthy blood donors. Thromb Haemost. 1994;71(1):68-72.

DECLARACIÓN DE CONFLICTO DE INTERESES

El autor declara que no existen conflictos de intereses. 